\title{
Relationships among the Hexosamine Biosynthetic Pathway, Sleep Disturbance, and Cognition in Obstructive Sleep Apnea
}

\author{
Ha Young Lee', Seong Min Oh², Mi Hyun Lee ${ }^{3}$ Jeong Eun Jeon', Kyung Hwa Lee', \\ Sunhee Jung ${ }^{4}$, Inn-Oc Han ${ }^{5}$, Geum-Sook Hwang ${ }^{4,6}$, and Yu Jin Lee ${ }^{1}$ \\ 'Department of Psychiatry and Center for Sleep and Chronobiology, Seoul National University College of Medicine, \\ Seoul National University Hospital, Seoul, Korea \\ ${ }^{2}$ Department of Psychiatry, Seoul National University College of Medicine, Seoul, Korea \\ ${ }^{3}$ Kosleep Sleep Clinic, Seoul, Korea \\ ${ }^{4}$ Integrated Metabolomics Research Group, Western Seoul Center, Korea Basic Science Institute, Seoul, Korea \\ ${ }^{5}$ Department of Biomedical Science, Program in Biomedical Science and Engineering, College of Medicine, Inha University, Incheon, Korea \\ ${ }^{6}$ Department of Chemistry and Nano Science, Ewha Womans University, Seoul, Korea
}

\begin{abstract}
Objective: This preliminary study aims to investigate the relationships among objective sleep variables, cognitive function, and hexosamine biosynthesis pathway (HBP) activation levels in middle- and older-aged adults with/without obstructive sleep apnea (OSA). Methods: A twenty-four participants (16 males and 8 females; mean age, 58.63 \pm .61 years) were recruited. The comprehensive attention test (CAT), peripheral blood collection, actigraphy (AG) and nocturnal polysomnography (nPSG) tests were conducted to evaluate cognitive function and the activation of HBP and sleep variables, respectively. Results: Total sleep time (TST) on AG was correlated positively with number of errors and mean response time (RT) on CAT. Sleep efficiency (SE) on AG was correlated negatively with number of errors and positively with mean RT, whereas the number of awakenings (NOA) was inversely associated with these variables. TST and SE on NPSG, SE on AG were positively correlated with levels of hexosamine pathway metabolites, whereas wake after sleep onset and sleep latency (SL) on nPSG, SL and NOA on AG were negatively correlated therewith. There was a negative correlation between levels of hexosamine pathway metabolites and number of errors on CAT. Conclusion: Sleep variables, cognitive function (i.e., attention), and HBP activation level were significantly correlated in middleand older-aged OSA participants.
\end{abstract}

Key Words: Obstructive sleep apnea; Cognition; Hexosamines

Received: June 11, 2021 Revised: June 17, 2021 Accepted: June 17, 2021

Corresponding author: Yu Jin Lee, MD, PhD, Department of Psychiatry and Center for Sleep and Chronobiology, Seoul National University College of Medicine and Seoul National University Hospital, 101 Daehak-ro, Jongno-gu, Seoul 03080, Korea.

Tel: 82-2-2072-0100, Fax: 82-2-766-1592, E-mail: ewpsyche@snu.ac.kr

(a) This is an Open Access article distributed under the terms of the Creative Commons Attribution Non-Commercial License (https://creativecommons.org/licenses/by$\mathrm{nc} / 4.0)$ which permits unrestricted non-commercial use, distribution, and reproduction in any medium, provided the original work is properly cited.

\section{INTRODUCTION}

Sleep occupies about a third of our lives and is essential for health and well-being [1]. Sleep disturbance adversely affects physiological, psychological, and cognitive functions. Obstructive sleep apnea (OSA) is a common sleep related breathing disorder characterized by intermittent upper-airway collapse during sleep that affects about $10 \%-20 \%$ of middle- and older-aged adults [2-4]. OSA makes it difficult to fall or stay asleep, resulting in poor sleep quality and sleep fragmentation [5], possibly pro- moting cognitive decline and neurodegeneration [6-8]. In zebrafish, hypoxia, a hallmark of OSA, induced cognitive impairment $[9,10]$. However, the mechanism by which sleep disorders cause cognitive dysfunction is unclear.

The hexosamine biosynthesis pathway (HBP) is active in most eukaryotic cells, accounts for $2-5 \%$ of glucose metabolism in humans, and causes intracellular protein modifications $[11,12]$. Olinked $\mathrm{N}$-acetylglucosamine $(\mathrm{O}-\mathrm{GlcNAc})$ is attached to intracellular proteins via UDP- $N$-acetylglucosamine (UDP-GlcNAc), the final metabolite of HBP, and this modification (i.e., O-GlcNAcyl- 
ation) increases protein stability and prevents protein aggregation [13]. Individuals with sleep disturbance and its risk factors (e.g., sleep deprivation, OSA, shift work) tend to show increased reactive oxygen species (ROS) levels [14-16]. ROS increase insulin resistance and decrease intracellular glucose levels, leading to reduction of HBP activation and O-GlcNAcylation [17]. There is also a close relationship between sleep disorders, including OSA, and increased insulin resistance $[18,19]$.

Decreased HBP activation may play an important role in cognitive impairment with aging. For example, $O$-GlcNAcylation has effects on many diseases related to aging, including diabetes, cardiomyopathies, and cancer [20]. It has been hypothesized that reduced $O$-GlcNAcylation in the brain causes aggregation of tau protein and accumulation of $\beta$-amyloid, which are associated with Alzheimer's disease [21]. In addition, patients with mild cognitive impairment showed low glucose metabolism in the brain [22], and glucose in the brain blood flow decreased with aging in normal controls [23]. Furthermore, HBP activation is likely involved in the associations of various sleep disorders with cognitive dysfunction. A zebrafish study found not only learning and memory impairments but also suppressed O-GlcNAcylation after sleep deprivation [24]. To summarize, sleep disturbance may affect insulin resistance and glucose metabolism, which could inhibit HBP and ultimately contribute to reduced brain function. Thus, it is possible that HBP activation levels mediate the relationship between sleep variables and cognitive function.

We compared the HBP activation of OSA and normal control (NC) groups and investigated the relationships among sleep parameters, HBP activation level, and cognitive function in OSA patients. To the best of our knowledge, few studies have investigated the relationships among these factors in people with/without OSA. Therefore, we recruited middle- and older-aged adults and analyzed sleep parameters using objective devices [i.e., nocturnal polysomnography (nPSG) and actigraphy (AG)], HBP activation data, and cognitive function as assessed by the comprehensive attention test (CAT). We aimed to identify the mechanism by which sleep disturbance causes cognitive decline and to provide evidence for the importance of treating sleep disorders.

\section{METHODS}

\section{Participants}

Sixteen males (mean age $59.69 \pm 8.56$ years, range $42-74$ years) and eight females (mean age $56.50 \pm 5.04$ years, range $51-65$ years) were recruited from advertisements or following an outpatient visit to Seoul National University Hospital. All participants were able to understand the explanation of the experiment and had a clinical dementia rating of 0 . The exclusion criteria were: major mental illnesses other than dementia, such as schizophrenia, bipolar disorder, major depression, alcohol/substance abuse or dependence, or delirium; neurological or medical disease that affects mental state; illiteracy sufficient to interfere with test performance; impairment of vision or hearing sufficient to interfere with test performance; participant in a drug trial; and other sleep disorders. This study was approved by the Institutional Review Board of Seoul National University Hospital (approval no. 1806-167-954), and all procedures were conducted after obtaining written informed consent from participants.

\section{Materials}

Participants' demographic information including age, sex, usual sleep habits, medical history, and drug use were recorded, followed by completion of the CAT and peripheral blood collection. Participants wore an AG on their non-dominant wrist for approximately 1 week and subsequently underwent one-night of nPSG.

\section{CAT}

We conducted the CAT, a computer-based test standardized in Korea, to evaluate cognitive function [25]. Participants conducted two types of tests measuring different attention abilities: the inhibition-sustained attention test (ISAT) and the divided-attention test (DAT). Inhibition-sustained attention is the maintenance or suppression of a response to repeated stimuli or actions. To evaluate whether the inhibition of a specific stimulus was maintained, participants were instructed to respond by pressing the space bar whenever various stimuli (e.g., circles, triangles, squares) appeared on a display, except for one stimulus (i.e., X-shape). Divided attention is the processing of more than one stimulus or piece of information simultaneously. To evaluate their ability to deal with successive stimuli presented simultaneously, participants were instructed to respond by pressing the space bar whenever they judged that the same stimulus as in the previous trial had been presented, irrespective of the stimulus type (e.g., visual stimuli: circles, triangles, and squares; auditory stimuli: bell sound and beep).

All participants performed the test after sufficient practice to learn the rules. The test time was 10 minutes for ISAT and $3 \mathrm{~min}$ utes and 20 seconds for DAT. The following indicators were measured in each test: commission error (CE; the number of wrong responses to a non-target stimulus), omission error (OE; the number of misses without responding to the target stimulus), mean response time of correct responses (mean RT), and sensitivity coefficient (d'; an indicator of how well the target stimulus and the non-target stimulus are distinguished).

\section{Quantitative analysis of metabolites by liquid chromatography-mass spectrometry}

To evaluate HBP activation, approximately $10 \mathrm{~mL}$ of blood were collected from the peripheral veins of the participants by trained experts. For targeted profiling of hexosamine pathway metabolites, $50 \mu \mathrm{L}$ of serum was mixed with $500 \mu \mathrm{L}$ of chloroform/methanol $(2: 1, \mathrm{v} / \mathrm{v})$ and $100 \mu \mathrm{L}$ of water. The mixture was vortexed and incubated at $4^{\circ} \mathrm{C}$ for 10 minutes. After centrifugation, $200 \mu \mathrm{L}$ of the supernatant was dried using $\mathrm{N}_{2}$ gas. The extracts were diluted with $50 \mu \mathrm{L}$ of an acetonitrile/water mixture (20:80, v/v). To quantify the levels of metabolites, liquid chromatography-mass spec- 
trometry (LC/MS) was performed on an Agilent 1290 Infinity LC and an Agilent 6495 Triple Quadrupole MS system equipped with an Agilent Jet Stream ESI source (Agilent Technologies, Santa Clara, CA, USA). MassHunter Workstation (ver. B.06.00, Agilent Technologies) software was used for data acquisition and analysis. LC separation was carried out on an Intrada amino acid column (150 mm×3 mm, Imtakt, Portland, OR, USA). The binary gradient system comprised $0.3 \%$ formic acid in $\mathrm{ACN}$ (solvent A) and $100 \mathrm{mM}$ ammonium formate in a mixture of $\mathrm{ACN}$ and water $(20: 80, v / v$, solvent $B)$. The linear gradients used for elution and equilibrating the initial gradient for subsequent runs were as follows: $0-27 \%$ B at $0-3$ minutes, $27-100 \%$ B at 3-19.5 minutes, $100 \%$ $\mathrm{B}$ at $19.5-25$ minutes, and $0 \% \mathrm{~B}$ at $25-32$ minutes. The column temperature and flow rate were $25^{\circ} \mathrm{C}$ and $0.6 \mathrm{~mL} /$ minute, respectively. The autosampler temperature was maintained at $10^{\circ} \mathrm{C}$, and the sample injection volume was $5 \mu \mathrm{L}$. Samples were analyzed in single reaction monitoring (SRM) positive ion mode. The SRM transitions were performed with the following operational parameters: capillary voltage, $2 \mathrm{kV}$; cone voltage, $20 \mathrm{~V}$; source temperature, $150^{\circ} \mathrm{C}$; desolvation temperature, $650^{\circ} \mathrm{C}$; cone gas flow, $150 \mathrm{~L} /$ hour; desolvation gas flow, $900 \mathrm{~L} /$ hour; collision gas flow, $0.15 \mathrm{~mL} /$ minute; and nebulizer gas flow, 7 bar. Concentrations of metabolites were quantified based on a series of external calibration curves generated using authentic standards. As a result of LCMS analysis, fructose-6-phosphate (F6P) and glucosamine-6-phosphate (G6P), $N$-acetyl glucosamine-1-phosphate (GlcNAc1P) and $N$-acetyl glucosamine-6-phosphate (GlcNAc6P), and UDPGlcNAc were quantified. Two substrates were reported as one value if isomeric metabolites were not well separated (i.e., F6P and G6P, GlcNAc1P and GlcNAc6P).

AG

AG is an objective sleep variable measurement method with high reliability and validity that is used to assess the circadian rhythm [26]. Participants wore an AG (Actiwatch-2, Philips Respironics, Cambridge, MA, USA) on their non-dominant wrist for about 1 week. Data were stored in 30-second epochs. Total sleep time (TST), sleep latency (SL), sleep efficiency (SE; defined as the ratio between TST and time spent in bed), wake after sleep onset (WASO), and number of awakenings (NOAs) were analyzed using the $\mathrm{AG}$ activation data.

\section{nPSG}

nPSG was performed to evaluate objective sleep parameters and exclude other sleep disorders. We used Neuvo system and Profusion EEG software (ver. 3.4; Compumedics, Abbotsford, VIC, Australia) for nPSG recordings in a standard manner and continuously measured electroencephalogram, electrooculogram, electrocardiogram, chin and limbs electromyogram, oral and nasal airflow, snoring sounds, abdominal movement, chest and abdominal movement, body position, and arterial oxygen saturation during night. The TST, WASO, SL, SE, apnea-hypopnea index (AHI), and periodic limb movement index (PLMI) were analyzed.
The sleep stages and events during sleep were scored and sleep parameters were calculated based on the American Academy of Sleep Medicine (AASM) criteria [27].

\section{Statistical analysis}

Data were analyzed using SPSS (ver. 25, IBM Corp., Armonk, NY, USA). The Mann-Whitney U-test was conducted to investigate differences in variables between the OSA and NC groups because the data failed to meet the normality assumption. Spearman's rho correlation analysis was performed to analyze the relationships among the intermediate and final hexosamine pathway metabolites, sleep parameters, and cognitive function indicators in participants with symptoms of OSA. Mediation analysis was performed with the PROCESS macro [28]. Statistical significance was accepted at $\mathrm{p}<0.05$.

\section{RESULTS}

\section{Demographic and baseline clinical characteristics}

We divided participants into a severe OSA group (30 $\mathrm{AHI})$ and an age-matched $\mathrm{NC}$ group (AHI $\leq 15)$ for the group comparison. Among all participants, six whose age did not match well or whose sleep apnea severity was not clearly classified were excluded from the group comparison. Nineteen participants diagnosed with OSA $(5 \leq \mathrm{AHI})$ were included in the correlation and mediation analysis. The demographic characteristics of the participants are listed in Table 1.

\section{Comparison of variables between the OSA and NC groups}

Table 2 shows the differences in objective sleep variables, levels of hexosamine pathway metabolites, and CAT results between the OSA and NC groups. The results, except for AHI, average and minimum $\mathrm{O} 2$ saturationm, and PLMI were not significant $(\mathrm{p}>0.05)$.

\section{Correlation analysis of sleep parameters with cognitive performance}

A Spearman's correlation analysis between sleep variables and CAT results was performed in the OSA patients (Table 3). No significant correlation of nPSG variables was observed $(\mathrm{p}>0.05)$. Regarding AG variables, TST was positively correlated with $\mathrm{OE}(\mathrm{r}=$ $0.67, \mathrm{p}<0.05)$ and the mean RT of ISAT $(\mathrm{r}=0.67, \mathrm{p}<0.05)$. SE was negatively correlated with the CE of ISAT $(r=-0.82, p<0.01)$ and positively correlated with the mean RT of ISAT and DAT (ISAT: $\mathrm{r}=0.92, \mathrm{p}<0.01$, DAT: $\mathrm{r}=0.82, \mathrm{p}<0.01)$. NOA had a positive correlation with $\mathrm{CE}(\mathrm{r}=0.92, \mathrm{p}<0.01)$ and a negative correlation with the mean RT of ISAT $(r=-0.80, \mathrm{p}<0.01)$.

\section{Correlation analysis of sleep parameters with HBP pathway metabolites}

A Spearman's correlation analysis between sleep variables and hexosamine pathway metabolites was performed in the OSA patients (Table 4). Regarding nPSG variables, TST had a positive cor- 
Table 1. Demographic and baseline clinical characteristics of the participants

\begin{tabular}{|c|c|c|c|c|}
\hline & & & Group & $1=18)$ \\
\hline & All $(n=24)$ & $5 \leq \mathrm{AHI}$ & $\begin{array}{c}\text { OSA }(n=9) \\
30 \leq \mathrm{AHI}\end{array}$ & $\begin{array}{c}\mathrm{NC}(\mathrm{n}=9) \\
\mathrm{AHI} \leq 15\end{array}$ \\
\hline Demographic & & & & \\
\hline Age (yrs) & $58.63 \pm 7.61$ & $60.37 \pm 7.17$ & $61.89 \pm 7.74$ & $59.00 \pm 6.19$ \\
\hline Male & $59.69 \pm 8.56$ & $61.36 \pm 7.42$ & $62.29 \pm 8.22$ & $63.20 \pm 4.35$ \\
\hline Female & $56.50 \pm 5.04$ & $57.60 \pm 6.31$ & $60.50 \pm 0.50$ & $53.75 \pm 1.79$ \\
\hline Sex & & & & \\
\hline Male & $16(66.67)$ & $14(73.68)$ & $7(77.78)$ & $5(55.56)$ \\
\hline Female & $8(33.33)$ & $5(26.32)$ & $2(22.22)$ & $4(44.44)$ \\
\hline Hexosamine pathway metabol & & & & \\
\hline F6P, G6P & $2,076.80 \pm 1,142.61$ & $2,116.01 \pm 1,254.59$ & $2,064.19 \pm 1,288.49$ & $2,089.41 \pm 1,055.35$ \\
\hline GlcNAc1P, GlcNAc6P & $637.36 \pm 190.04$ & $607.31 \pm 189.19$ & $592.66 \pm 201.19$ & $682.06 \pm 178.26$ \\
\hline UDP-GlcNAc & $4,585.85 \pm 2,986.18$ & $4,804.30 \pm 3,067.51$ & $4,557.11 \pm 3,151.15$ & $4,614.60 \pm 3,002.93$ \\
\hline Sleep parameters & & & & \\
\hline nPSG & & & & \\
\hline TST & $384.10 \pm 55.79$ & $371.63 \pm 55.80$ & $348.83 \pm 65.68$ & $401.83 \pm 43.03$ \\
\hline SE & $5.75 \pm 4.92$ & $6.08 \pm 5.11$ & $82.89 \pm 12.07$ & $89.06 \pm 5.55$ \\
\hline SL & $86.51 \pm 9.47$ & $85.17 \pm 10.18$ & $5.17 \pm 4.81$ & $4.28 \pm 3.02$ \\
\hline WASO & $53.40 \pm 41.46$ & $58.21 \pm 44.91$ & $66.11 \pm 50.53$ & $43.83 \pm 24.38$ \\
\hline AHI & $21.43 \pm 18.84$ & $26.25 \pm 18.32$ & $42.88 \pm 12.47$ & $8.46 \pm 4.77$ \\
\hline Average $\mathrm{O}_{2}$ saturation & $93.79 \pm 2.41$ & $93.47 \pm 2.61$ & $91.22 \pm 1.92$ & $95.22 \pm 0.97$ \\
\hline Minimum $\mathrm{O}_{2}$ saturation & $84.13 \pm 7.23$ & $82.42 \pm 7.15$ & $77.89 \pm 7.59$ & $88.00 \pm 4.15$ \\
\hline PLMI & $7.68 \pm 16.55$ & $8.32 \pm 18.22$ & $1.66 \pm 2.67$ & $17.57 \pm 24.33$ \\
\hline Actigraphy & & & & \\
\hline TST & $426.75 \pm 118.65$ & $411.62 \pm 102.84$ & $382.17 \pm 57.51$ & $482.71 \pm 156.34$ \\
\hline SE & $18.21 \pm 15.00$ & $18.36 \pm 15.84$ & $79.36 \pm 10.73$ & $85.97 \pm 7.50$ \\
\hline SL & $81.99 \pm 8.73$ & $81.28 \pm 8.18$ & $14.39 \pm 11.38$ & $13.28 \pm 9.49$ \\
\hline WASO & $55.84 \pm 36.03$ & $59.09 \pm 38.23$ & $73.37 \pm 55.21$ & $44.12 \pm 14.37$ \\
\hline $\mathrm{NOA}$ & $42.80 \pm 16.18$ & $46.34 \pm 14.75$ & $48.54 \pm 19.58$ & $35.15 \pm 13.67$ \\
\hline CAT & & & & \\
\hline Inhibition-sustained attentic & & & & \\
\hline $\mathrm{CE}$ & $5.44 \pm 60$ & $5.54 \pm 6.46$ & $1.20 \pm 2.68$ & $3.29 \pm 4.03$ \\
\hline $\mathrm{OE}$ & $2.31 \pm 3.20$ & $2.23 \pm 3.54$ & $5.60 \pm 7.44$ & $3.43 \pm 3.15$ \\
\hline RT & $497.11 \pm 83.03$ & $499.39 \pm 85.32$ & $490.18 \pm 57.79$ & $542.52 \pm 87.59$ \\
\hline$d^{\prime}$ & $4.05 \pm 0.78$ & $4.09 \pm 0.81$ & $4.33 \pm 1.06$ & $4.06 \pm 0.73$ \\
\hline Divided attention test & & & & \\
\hline $\mathrm{CE}$ & $4.50 \pm 5.28$ & $3.23 \pm 1.54$ & $13.00 \pm 14.20$ & $15.71 \pm 14.52$ \\
\hline $\mathrm{OE}$ & $13.44 \pm 12.09$ & $12.92 \pm 10.86$ & $3.40 \pm 1.67$ & $5.57 \pm 8.06$ \\
\hline RT & $757.97 \pm 143.4$ & $768.94 \pm 132.46$ & $741.35 \pm 79.11$ & $797.96 \pm 191.65$ \\
\hline$d^{\prime}$ & $2.20 \pm 0.60$ & $2.28 \pm 0.55$ & $2.34 \pm 0.86$ & $2.09 \pm 0.61$ \\
\hline
\end{tabular}

Data are presented as mean \pm standard deviation or $\mathrm{n}(\%)$. OSA: obstructive sleep apnea, F6P: fructose 6-phosphate, G6P: glucose 6-phosphate, GlcNAc1P: $N$-acetyl- $D$-glucosamine 1-phosphate, GlcNAc6P: $N$-acetylglucosamine-6-phosphate, UDP-GlcNAc: uridine diphosphate $N$-acetylglucosamine, nPSG: nocturnal polysomnography, TST: total sleep time, SE: sleep efficiency, WASO: wake after sleep onset, SL: sleep latency, AHI: apnea hypopnea index, PLMI: periodic limb movement index, NOA: number of awakenings, CAT: comprehensive attention test, CE: comission errors, OE: omission errors, RT: mean response time of correct responses, d': sensitivity coefficient

relation with levels of UDP-GlcNAc $(\mathrm{r}=0.65, \mathrm{p}<0.01)$. SL was negatively correlated with F6P and G6P, GlcNAc1P and GlcNAc6P (F6P and G6P; r=-0.70, p<0.01; GlcNAc1P and GlcNAc6P: $r=$ $-0.64, \mathrm{p}<0.05)$. SE was positively correlated with GlcNAc1P and GlcNAc6P, and UDP-GlcNAc (GlcNAc1P and GlcNAc6P: r=0.59, $\mathrm{p}<0.05$; UDP-GlcNAc: $\mathrm{r}=0.65, \mathrm{p}<0.01)$. WASO was negatively correlated with UDP-GlcNAc $(\mathrm{r}=-0.61, \mathrm{p}<0.05)$. In terms of AG variables, SL was negatively correlated with GlcNAc1P and GlcNAc6P $(\mathrm{r}=-0.69, \mathrm{p}<0.05)$. SE was positively correlated with the levels of GlcNAc1P and GlcNAc6P $(\mathrm{r}=0.72, \mathrm{p}<0.05)$. NOA was negatively correlated with GlcNAc1P and GlcNAc6P $(\mathrm{r}=-0.75, \mathrm{p}<0.05)$.

Correlation analysis of HBP pathway metabolites with cognitive performance

A Spearman's correlation analysis of hexosamine pathway metabolites and CAT results was performed in the OSA patients (Ta- 
Table 2. Differences in variables between the OSA and NC groups $(n=18)$

\begin{tabular}{|c|c|c|c|}
\hline & $\mathrm{U}$ & $\mathrm{Z}$ & $\mathrm{p}$ \\
\hline \multicolumn{4}{|c|}{ Hexosamine pathway metabolites } \\
\hline F6P, G6P & 39.00 & -0.13 & 0.89 \\
\hline GlcNAc1P, GlcNAc6P & 28.00 & -1.10 & 0.27 \\
\hline UDP-GlcNAc & 40.00 & -0.04 & 0.96 \\
\hline \multicolumn{4}{|l|}{ Sleep parameters } \\
\hline \multicolumn{4}{|l|}{ nPSG } \\
\hline TST & 21.50 & -1.68 & 0.09 \\
\hline SE & 29.00 & -1.02 & 0.31 \\
\hline SL & 39.00 & -0.13 & 0.89 \\
\hline WASO & 35.00 & -0.49 & 0.63 \\
\hline AHI & 0.00 & -3.58 & $<0.001^{\dagger}$ \\
\hline Average $\mathrm{O}_{2}$ saturation & 4.00 & -3.27 & $0.001^{\dagger}$ \\
\hline Mininum $\mathrm{O}_{2}$ saturation & 9.00 & -2.79 & $0.005^{\dagger}$ \\
\hline PLMI & 14.00 & -2.45 & $0.01^{*}$ \\
\hline \multicolumn{4}{|l|}{ Actigraphy } \\
\hline TST & 12.00 & -1.29 & 0.20 \\
\hline SE & 13.00 & -1.14 & 0.25 \\
\hline SL & 19.00 & -0.29 & 0.78 \\
\hline WASO & 17.00 & -0.57 & 0.57 \\
\hline NOA & 11.00 & -1.43 & 0.15 \\
\hline \multicolumn{4}{|l|}{ CAT } \\
\hline \multicolumn{4}{|c|}{ Inhibition-sustained attention test } \\
\hline $\mathrm{CE}$ & 17.00 & -0.08 & 0.93 \\
\hline $\mathrm{OE}$ & 8.00 & -1.61 & 0.11 \\
\hline RT & 10.00 & -1.22 & 0.22 \\
\hline $\mathrm{d}^{\prime}$ & 13.50 & -0.65 & 0.52 \\
\hline \multicolumn{4}{|l|}{ Divided attention test } \\
\hline CE & 17.00 & -0.08 & 0.93 \\
\hline $\mathrm{OE}$ & 13.50 & -0.65 & 0.52 \\
\hline RT & 15.00 & -0.41 & 0.68 \\
\hline$d^{\prime}$ & 12.50 & -0.81 & 0.42 \\
\hline
\end{tabular}

${ }^{*} \mathrm{p}<0.05,{ }^{\dagger} \mathrm{p}<0.01$. OSA: obstructive sleep apnea, F6P: fructose 6-phosphate, G6P: glucose 6-phosphate, GlcNAc1P: $N$-acetyl- $D$-glucosamine 1-phosphate, GlcNAc6P: N-acetylglucosamine-6-phosphate, UDPGlcNAc: uridine diphosphate $N$-acetylglucosamine, nPSG: nocturnal polysomnography, TST: total sleep time, SE: sleep efficiency, WASO: wake after sleep onset, SL: sleep latency, AHI: apnea hypopnea index, PLMI: periodic limb movement index, NOA: number of awakenings, CAT: comprehensive attention test, CE: comission errors, OE: omission errors, RT: mean response time of correct responses, d': sensitivity coefficient

ble 5). The GlcNAc1P and GlcNAc6P levels were negatively correlated with CE of ISAT $(\mathrm{r}=-0.71, \mathrm{p}<0.05)$.

\section{Mediating effect of HBP activation between sleep parameters and cognitive performance}

A mediation analysis was performed to determine whether the levels of hexosamine pathway metabolites mediated the relationship between sleep variables and CAT results. We tested two models using significantly correlated variables for both all participants and the OSA symptom group. In the first model, SE measured by AG was used as the independent variable, CE of ISAT as a depen- dent variable, and GlcNAc1P and GlcNAc6P as mediator variables. In the second model, NOA measured by AG was used as the independent variable, CE of ISAT as a dependent variable, and GlcNAc1P and GlcNAc6P as mediator variables. No significant mediating effect was observed ( $p>0.05)$.

\section{DISCUSSION}

In the current study, we examined the associations between objective sleep variables, CAT results, and the levels of hexosamine pathway metabolites in blood, to investigate the relationships among sleep disturbances, cognitive function, and HPB activation in middle- and older-aged adults with/without OSA. Sleep apnea is linked to alterations in, and may suppress, glucose metabolism $[29,30]$. Regarding the underlying mechanism, Punjabi and Polotsky [31] conjectured that sleep fragmentation and hypoxemia during sleep apnea alter the hypothalamic-pituitaryadrenal and somatotropic axis, thus affecting glucose tolerance. However, we observed no significant difference of hexosamine pathway metabolites between the OSA and NC groups. The reason for this might be the small sample size and/or ethical limitations of human studies. Whereas prior animal studies involved extreme hypoxic conditions [24,32], in this human study, OSA patients experiencing intermittent hypoxia were recruited. We classified participants into a severe OSA group and a relatively healthy NC group, but the experimental conditions might not have been sufficient to produce the expected difference.

Participants who slept longer missed more responses (i.e., OE) and took longer to provide the correct answer. Since there was no significant correlation with $\mathrm{CE}$, it may just have been that they exceeded the given time to respond carefully. Those who slept with high efficiency responded better to target stimuli and took longer to provide the correct answer, and those who woke less frequently during sleep responded better to target stimuli. Given that mean RT and CE revealed opposite correlations, it can be supposed that individuals who have relatively low sleep quality might tend to respond more impulsively in cognitive judgments. This is supported by previous reports of significant associations between impulsivity and shorter TST and lower SE as measured by AG [33], and shorter sleep duration based on self-reported questionnaires [34]. However, because cognitive change usually occurs over a long period and this was not a longitudinal study, the relationship needs to be confirmed.

Participants who slept less, took more time to fall asleep, had less efficient sleep, and were awake for more time and more often during sleep had lower levels of HBP metabolites. Also, those with lower levels of HBP metabolites made fewer errors in cognitive tests. Alzheimer's disease, which is accompanied by sleep disturbance, results in low UDP-GlcNAc levels [21,35]. Lee et al. [24] reported that $O$-GlcNAcylation in the zebrafish brain decreased as sleep deprivation time increased, demonstrating impaired learning and memory effects. That result implied that sleep disturbance precedes metabolic changes and cognitive decline. 


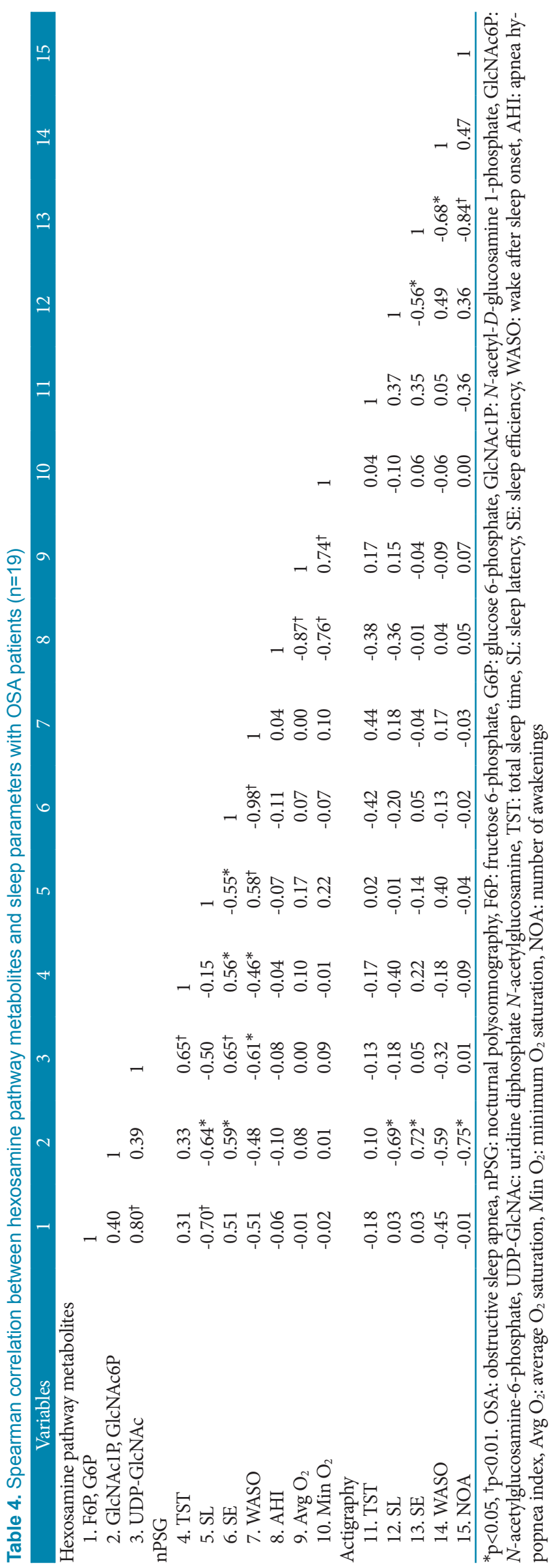

Based on the prior studies, we expected that levels of metabolites would have a mediating effect between sleep variables and cognitive performance. However, our testing of models in which each variable showed significant correlations indicated that the mediating effects were not significant. This might have been because the number of samples was not sufficient to perform a mediation analysis.

\section{Limitations}

This study had several limitations. First, the sample size was small. The study should be replicated with a larger number of participants to verify whether the small sample size affected significance. Second, the correlations and cross-sectional design cannot explain causality or directionality between variables. Third, we used only attention tests as a measure of cognitive function and conducted them only once. A further study needs to evaluate longer-term cognitive tests. Fourth, sleep may not have been accurately assessed. One-night nPSG is the most accurate test for measuring sleep parameters by EEG, but does not always represent those of usual sleep. AG is assumed to represent sleep over 7-10 days, although it is less accurate. We used both to obtain more accurate results. Fifth, it is not clear whether blood collected from peripheral veins reflects molecular changes in the brain. Previous studies used the brains of deceased patients or animals to analyze levels of hexosamine pathway metabolites. This methodological difference may have affected the results. Finally, the prevalence of OSA in this study was high since we recruited middle-aged and older adults to examine the associations between sleep, cognition, and HBP through in-hospital posters or outpatients. Therefore, in order to match the number of the severe OSA group, the criteria of the $\mathrm{NC}$ group were defined more generously $(\mathrm{AHI} \leq 15)$ than the clinical criteria $(\mathrm{AHI}<5)$. Future studies should be designed in consideration of the points.

\section{Conclusion}

This is the first study to directly investigate the associations among objective sleep variables, HBP activation, and cognitive function in both OSA patients and healthy controls. The OSA and $\mathrm{NC}$ groups showed no significant differences.

Sleep parameters, cognitive function, and HBP activation level showed significant relationships. Participants with high sleep quality generally responded more slowly, gave more correct answers in the cognitive test, and had higher levels of hexosamine pathway metabolites. Those who had higher levels of metabolites made fewer errors in the cognitive test. There was no significant mediating effect of HBP activation on the relationship between sleep and cognitive ability. Nevertheless, this study is meaningful in that we attempted to replicate prior animal experiments in middle- and older-aged humans and suggested directions for followup research. Such studies will enable quantitative evaluation of the effects of sleep disorders on HBP activation and cognitive decline and the development of novel treatments. 
Table 5. Spearman correlation between hexosamine pathway metabolites and cognitive variables with OSA patients ( $\mathrm{n}=19$ )

\begin{tabular}{|c|c|c|c|c|c|c|c|c|c|c|c|}
\hline Variables & 1 & 2 & 3 & 4 & 5 & 6 & 7 & 8 & 9 & 10 & 11 \\
\hline \multicolumn{12}{|c|}{ Hexosamine pathway metabolites } \\
\hline 1. F6P, G6P & 1 & & & & & & & & & & \\
\hline 2. GlcNAc1P, GlcNAc6P & 0.40 & 1 & & & & & & & & & \\
\hline 3. UDP-GlcNAc & $0.80^{\dagger}$ & 0.39 & 1 & & & & & & & & \\
\hline \multicolumn{12}{|c|}{ Inhibition-sustained attention test } \\
\hline 4. $\mathrm{CE}$ & 0.07 & $-0.71^{*}$ & -0.02 & 1 & & & & & & & \\
\hline 5. OE & -0.07 & -0.18 & 0.13 & 0.34 & 1 & & & & & & \\
\hline 6. RT & -0.15 & 0.47 & -0.10 & $-0.78^{\dagger}$ & -0.08 & 1 & & & & & \\
\hline 7. d' & -0.03 & 0.61 & -0.01 & $-0.85^{\dagger}$ & $-0.71^{\dagger}$ & 0.54 & 1 & & & & \\
\hline \multicolumn{12}{|l|}{ Divided attention test } \\
\hline 8. CE & 0.26 & 0.27 & -0.15 & 0.19 & -0.10 & -0.18 & -0.16 & 1 & & & \\
\hline 9. OE & -0.23 & -0.22 & 0.07 & 0.28 & 0.47 & -0.28 & -0.29 & $-0.71^{\dagger}$ & 1 & & \\
\hline 10. RT & -0.47 & 0.23 & -0.22 & -0.46 & 0.23 & 0.48 & 0.35 & -0.25 & 0.13 & 1 & \\
\hline 11. d' & -0.03 & 0.40 & -0.12 & -0.47 & -0.48 & 0.47 & 0.42 & 0.53 & $-0.94^{\dagger}$ & 0.02 & 1 \\
\hline
\end{tabular}

${ }^{*} \mathrm{p}<0.05,{ }^{\dagger} \mathrm{p}<0.01$. OSA: obstructive sleep apnea, F6P: fructose 6-phosphate, G6P: glucose 6-phosphate, GlcNAc1P: $N$-acetyl-D-glucosamine 1-phosphate, GlcNAc6P: $N$-acetylglucosamine-6-phosphate, UDP-GlcNAc: uridine diphosphate $N$-acetylglucosamine, CE: commission errors, OE: omission errors, RT: mean response time of correct responses, d': sensitivity coefficient

Acknowledgments

This study was supported by Seoul National University Hospital Research Fund (grant no.: 04-2018-246).

\section{Conflicts of Interest}

The authors have no potential conflicts of interest to disclose.

Author Contributions

Conceptualization: Yu Jin Lee, Inn-Oc Han. Data curation: Seong Min Oh, Mi Hyun Lee, Jeong Eun Jeon. Formal analysis: Ha Young Lee, Kyung Hwa Lee. Funding acquisition: Yu Jin Lee. Investigation: Yu Jin Lee, Sunhee Jung, Geum-Sook Hwang. Methodology: Seong Min Oh, Sunhee Jung, Geum-Sook Hwang. Supervision: Yu Jin Lee, Inn-Oc Han. Writing—original draft: Ha Young Lee, Seong Min Oh. Writing_review \& editing: Yu Jin Lee.

\section{ORCID iDs}

Ha Young Lee (1)

https://orcid.org/0000-0002-7769-400X

Seong Min Oh (1)

https://orcid.org/0000-0001-8895-8990

Mi Hyun Lee

https://orcid.org/0000-0003-0377-7555

Jeong Eun Jeon (1)

https://orcid.org/0000-0002-1723-0123

Kyung Hwa Lee (1)

https://orcid.org/0000-0002-6168-5432

Sunhee Jung (1)

https://orcid.org/0000-0001-5396-4813

Inn-Oc Han (10)

https://orcid.org/0000-0003-3000-8428

Geum-Sook Hwang (10

https://orcid.org/0000-0002-1600-1556
Yu Jin Lee

https://orcid.org/0000-0001-5195-2579

\section{REFERENCES}

1. Lee KA. An overview of sleep and common sleep problems. ANNA J 1997; 24:614-623, 677

2. Strollo PJ Jr, Rogers RM. Obstructive sleep apnea. N Engl J Med 1996;334: 99-104.

3. Young T, Skatrud J, Peppard PE. Risk factors for obstructive sleep apnea in adults. JAMA 2004;291:2013-2016.

4. Balk EM, Moorthy D, Obadan NO, Patel K, Ip S, Chung M, et al. Diagnosis and treatment of obstructive sleep apnea in adults. Comparative effectiveness review no. 32. Rockville, MD: Agency for Healthcare Research and Quality; 2011.

5. Ong JC, Crawford MR, Wallace DM. Sleep apnea and insomnia: emerging evidence for effective clinical management. Chest 2021;159:2020-2028.

6. Lim AS, Kowgier M, Yu L, Buchman AS, Bennett DA. Sleep fragmentation and the risk of incident Alzheimer's disease and cognitive decline in older persons. Sleep 2013;36:1027-1032.

7. Daulatzai MA. Evidence of neurodegeneration in obstructive sleep apnea: relationship between obstructive sleep apnea and cognitive dysfunction in the elderly. J Neurosci Res 2015;93:1778-1794.

8. Raven F, Van der Zee EA, Meerlo P, Havekes R. The role of sleep in regulating structural plasticity and synaptic strength: implications for memory and cognitive function. Sleep Med Rev 2018;39:3-11.

9. Lee Y, Lee S, Park JW, Hwang JS, Kim SM, Lyoo IK, et al. Hypoxia-induced neuroinflammation and learning-memory impairments in adult zebrafish are suppressed by glucosamine. Mol Neurobiol 2018;55:8738-8753.

10. Ramalingam L, Madhaiyan V. Hypoxia and its effects on learning and memory in a zebrafish model. Natl J Physiol Pharm Pharmacol 2020;10:955-960.

11. Wells L, Vosseller K, Hart GW. Glycosylation of nucleocytoplasmic proteins: signal transduction and O-GlcNAc. Science 2001;291:2376-2378.

12. Vosseller K, Sakabe K, Wells L, Hart GW. Diverse regulation of protein function by O-GlcNAc: a nuclear and cytoplasmic carbohydrate post-translational modification. Curr Opin Chem Biol 2002;6:851-857.

13. Sohn KC, Lee KY, Park JE, Do SI. OGT functions as a catalytic chaperone under heat stress response: a unique defense role of OGT in hyperthermia. Biochem Biophys Res Commun 2004;322:1045-1051.

14. Trivedi MS, Holger D, Bui AT, Craddock TJA, Tartar JL. Short-term sleep deprivation leads to decreased systemic redox metabolites and altered epigenetic status. PLoS One 2017;12:e0181978. 
15. Bhatti P, Mirick DK, Randolph TW, Gong J, Buchanan DT, Zhang JJ, et al. Oxidative DNA damage during night shift work. Occup Environ Med 2017; 74:680-683.

16. Snyder B, Shell B, Cunningham JT, Cunningham RL. Chronic intermittent hypoxia induces oxidative stress and inflammation in brain regions associated with early-stage neurodegeneration. Physiol Rep 2017;5:e13258.

17. Panigrahy SK, Bhatt R, Kumar A. Reactive oxygen species: sources, consequences and targeted therapy in type 2 diabetes. J Drug Target 2017;25:93101.

18. Murphy AM, Thomas A, Crinion SJ, Kent BD, Tambuwala MM, Fabre A, et al. Intermittent hypoxia in obstructive sleep apnoea mediates insulin resistance through adipose tissue inflammation. Eur Respir J 2017;49:1601731.

19. Deng HB, Tam T, Zee BC, Chung RY, Su X, Jin L, et al. Short sleep duration increases metabolic impact in healthy adults: a population-based cohort study. Sleep 2017;40:zsx130.

20. Banerjee PS, Lagerlöf O, Hart GW. Roles of O-GlcNAc in chronic diseases of aging. Mol Aspects Med 2016;51:1-15.

21. Yuzwa SA, Vocadlo DJ. O-GlcNAc and neurodegeneration: biochemical mechanisms and potential roles in Alzheimer's disease and beyond. Chem Soc Rev 2014;43:6839-6858.

22. Drzezga A, Lautenschlager N, Siebner H, Riemenschneider M, Willoch F, Minoshima S, et al. Cerebral metabolic changes accompanying conversion of mild cognitive impairment into Alzheimer's disease: a PET follow-up study. Eur J Nucl Med Mol Imaging 2003;30:1104-1113.

23. Heiss WD, Szelies B, Kessler J, Herholz K. Abnormalities of energy metabolism in Alzheimer's disease studied with PET. Ann N Y Acad Sci 1991;640: 65-71.

24. Lee Y, Park J, Kim SM, Nam K, Lee W, Cho H, et al. Sleep deprivation impairs learning and memory by decreasing protein O-GlcNAcylation in the brain of adult zebrafish. FASEB J 2020;34:853-864.

25. Huh HN, Kang SH, Hwang SY, Yoo HK. Developmental trajectories of at- tention in normal Korean population. J Korean Acad Child Adolesc Psychiatry 2019;30:66-73.

26. Teicher MH. Actigraphy and motion analysis: new tools for psychiatry. Harv Rev Psychiatry 1995;3:18-35.

27. Iber C. The AASM manual for the scoring of sleep and associated events: rules, terminology and technical specification. Westchester, IL: American Academy of Sleep Medicine; 2007.

28. Hayes AF. Introduction to mediation, moderation, and conditional process analysis: a regression-based approach. New York, NY: The Guilford Press; 2017.

29. Tasali E, Ip MS. Obstructive sleep apnea and metabolic syndrome: alterations in glucose metabolism and inflammation. Proc Am Thorac Soc 2008; 5:207-217.

30. Lindberg E, Theorell-Haglöw J, Svensson M, Gislason T, Berne C, Janson C. Sleep apnea and glucose metabolism: a long-term follow-up in a community-based sample. Chest 2012;142:935-942.

31. Punjabi NM, Polotsky VY. Disorders of glucose metabolism in sleep apnea. J Appl Physiol (1985) 2005;99:1998-2007.

32. Kim YH, Lee KS, Kim YS, Kim YH, Kim JH. Effects of hypoxic preconditioning on memory evaluated using the T-maze behavior test. Anim Cells Syst (Seoul) 2019;23:10-17.

33. McGowan NM, Coogan AN. Sleep and circadian rhythm function and trait impulsivity: an actigraphy study. Psychiatry Res 2018;268:251-256.

34. Zhu J, Jiang Y, Chen Y, Huang L, Bao Z, Zhang W. High impulsivity, low selfcontrol and problematic mobile phone use: the effect of poor sleep quality. Curr Psychol 2019 Apr 23 [Epub]. Available at: https://doi.org/10.1007/ s12144-019-00259-0.]

35. Liu F, Shi J, Tanimukai H, Gu J, Gu J, Grundke-Iqbal I, et al. Reduced OGlcNAcylation links lower brain glucose metabolism and tau pathology in Alzheimer's disease. Brain 2009;132:1820-1832. 